\title{
Exploring the canonical behaviour of long gamma-ray bursts with an intrinsic multi-wavelength afterglow correlation.
}

\section{S. R. Oates ${ }^{* a}$, J. L. Racusin ${ }^{b}$, M. De Pasquale ${ }^{c}$, D. Kocevski ${ }^{b}$, M. J. Page ${ }^{d}$, A. J.} Castro-Tirado $^{e f}$, J. Gorosabel ${ }^{e g h \dagger}$, A. A. Breeveld ${ }^{d}$, N. P. M. Kuin ${ }^{d}$ and P. J. Smith ${ }^{d}$

${ }^{a}$ Department of Physics, University of Warwick, Coventry, CV4 7AL, UK

${ }^{b}$ Astrophysics Science Division, NASA Goddard Space Flight Center, 8800 Greenbelt Road, Greenbelt, Maryland 20771, USA

${ }^{c}$ Department of Astronomy and Space Sciences, Istanbul University, Beyazit, 34119, Istanbul, Turkey

${ }^{d}$ Mullard Space Science Laboratory, University College London, Holmbury St. Mary, Dorking, Surrey, RH5 6NT, UK

e Instituto de Astrofísica de Andalucía (IAA-CSIC), Glorieta de la Astronomía s/n, E-18008, Granada, Spain

${ }^{f}$ Unidad Asociada Departamento de Ingeniería de Sistemas y Automática, E.T.S. de Ingenieros Industriales, Universidad de Málaga, Spain

${ }^{g}$ Unidad Asociada Grupo Ciencias Planetarias UPV/EHU-IAA/CSIC, Departamento de Física Aplicada I, E.T.S.

${ }^{h}$ Ingeniería, Universidad del País Vasco UPV/EHU, Bilbao, Spain

$\dagger$ deceased

E-mail: s.oates@warwick.ac.uk

In this conference proceeding we examine a correlation between the afterglow luminosity (measured at restframe $200 \mathrm{~s} ; \log \mathrm{L}_{200 \mathrm{~s}}$ ) and average afterglow decay rate (measured from restframe $200 \mathrm{~s}$ onwards; $\alpha>200 \mathrm{~s}$ ) found in both the optical/UV and X-ray afterglows of long duration Gamma-ray Bursts (GRBs). Examining the X-ray light curves, we find the correlation does not depend on the presence of specific light curve features. We explore how the parameters in the optical/UV and X-ray bands relate to each other and to the prompt emission phase. We also use a Monte Carlo simulation to explore whether these relationships are consistent with predictions of the standard afterglow model. We conclude that the correlations are consistent with a common underlying physical mechanism producing GRBs and their afterglows regardless of their detailed temporal behaviour. However, a basic afterglow model has difficulty explaining correlations involving $\alpha_{>200 s}$. We therefore briefly discuss alternative more complex models.

Multifrequency Behaviour of High Energy Cosmic Sources - XIII - MULTIF2019

3-8 June 2019

Palermo, Italy

${ }^{*}$ Speaker. 


\section{Introduction}

Gamma-ray bursts (GRBs) are intense flashes of gamma-rays that are usually accompanied by an afterglow; longer lived emission that may be detected at X-ray to radio wavelengths. Statistical investigations performed with GRBs so far have found a number of trends and correlations within and linking the prompt gamma-ray emission and the afterglow emission e.g, $[1,2,3,4,5,6,7,8$, $9,10]$. Within the afterglow, several trends are apparent and are being explored. One such example is a correlation between the logarithmic optical/UV brightness (log $\mathrm{L}_{\mathrm{O}, 200 \mathrm{~s}}$; measured at restframe $200 \mathrm{~s}$ and at a restframe wavelength $1600 \AA)$, and average decay rate of GRB afterglows $\left(\alpha_{>200 \mathrm{~s}}\right.$; measured from restframe $200 \mathrm{~s}$ onwards with a single power-law) [11]. This was discovered using a sample of 48 optical/UV GRB light curves observed by the Ultra-violet Optical Telescope (UVOT)[12] onboard the Neil Gehrels Swift Observatory (hereafter Swift) [13]. A Spearman rank test gave a coefficient of -0.58 at a significance of $99.998 \%(4.2 \sigma)$. This correlation suggests the brightest optical/UV afterglows decay more quickly on average compared to fainter optical/UV afterglows.

To gain insight into the origin of this correlation, we wished to determine if this correlation is observed also in the X-ray and how it relates to other GRB properties. We performed this analysis in [14] and [15]. [14] searched for a $\log L_{200 s}-\alpha_{>200 s}$ in the X-ray afterglows observed by the Swift X-ray Telescope (XRT; [16]). [15] compared the parameters of the optical/UV log $\mathrm{L}_{200 \mathrm{~s}}-$ $\alpha>200$ s correlation with the equivalent values from the X-ray and also explored their relationship to properties of the prompt emission, namely the isotropic energy $E_{\text {iso }}$ and the peak energy $E_{\text {peak }}$. We will provide a summary of these papers in this conference proceeding.

\section{Sample Selection and Data Analysis}

The sample of X-ray light curves consists of 237 Swift-BAT discovered GRBs with measured redshifts, discovered between December 2004 and March 2014 (for further details on sample selection see [14]). All light curves were retrieved from the University of Leicester Swift-XRT Team GRB repository $[17,18]$. The count rate light curves were converted to flux density at $1 \mathrm{keV}$ using the spectral index from the automated fits to the photon counting mode data, and then to intrinsic luminosity. All light curve fitting is performed in the count rate domain.

The optical/UV sample consists of 56 long duration GRBs, selected from the second Swift UVOT GRB afterglow catalogue [19], which were observed between April 2005 and December 2010. They were selected using the criteria of [20]. These criteria ensure that a high signal-to-noise (SN) light curve, covering both early and late times, could be constructed from the UVOT multifilter observations (see $[20,11]$ ). These GRBs have spectroscopic or photometric redshifts and we were able to determine the host $\mathrm{E}(\mathrm{B}-\mathrm{V})$ values (the host extinction was derived from afterglow spectral energy distributions following [21]). For each GRB, optical/UV luminosity light curves were produced at a common wavelength of $1600 \AA$ [11].

For each GRB, we interpolated the optical/UV luminosity at $200 \mathrm{~s}$ using data between 100 and $2000 \mathrm{~s}$ and for the X-ray we measured the luminosity at $200 \mathrm{~s}$ from the best-fit light curve model $[22,23]$. To obtain the average decay rate, we fit a single power-law to each optical/UV and X-ray light curve using data from $200 \mathrm{~s}$ onwards. For 8 optical/UV light curves, we were unable 
to determine the luminosity at $200 \mathrm{~s}$ and/or the average decay index. We therefore excluded these GRBs from the optical/UV sample.

An initial steep decay, associated with the tail of the prompt emission [24], for some GRBs is found to be contaminating the X-ray afterglows. For 8 of the $237 \mathrm{X}$-ray light curves the steep decay segment is found to contaminate the X-ray light curves at restframe $200 \mathrm{~s}$. We identify a light curve segment to have a prompt origin if there is a steep to shallow transition with $\Delta \alpha>1.0$. In these situations the average decay index is measured with a simple power-law fit to data beyond restframe $200 \mathrm{~s}$ and after the steep to shallow transition. In order to get a better estimate of the afterglow luminosity at restframe $200 \mathrm{~s}$, we extrapolate back to restframe $200 \mathrm{~s}$ the first segment of the best-fit light curve that is not contaminated by the prompt emission (see also [14]).

To compare the afterglow properties with the prompt emission properties we determined the isotropic energy $E_{\text {iso }}$ and restframe peak energy, $E_{\text {peak }}$ from the $\gamma$-ray emission, following [22]. Of the 48 GRBs in our optical/UV sample, we could determine $\mathrm{E}_{\text {peak }}$ for 44 and $\mathrm{E}_{\text {iso }}$ for 47.

In order to determine the strength and significance of each correlation, we perform a linear regression analysis using the IDL routines fitexy and sixlin: fitexy is used when both parameters have errors, sixlin is used when we do not know the errors on one or both parameters. Since there are only a handful of GRBs with errors on the $E_{\text {iso }}$ and $E_{\text {peak }}$ parameters, we choose to discard errors in both parameters and use sixlin when determining the strength and significance of each correlation with one of these parameters involved.

We determine the strength of the correlation by measuring a Spearman rank coefficient $\left(R_{s p}\right)$, and its corresponding null hypothesis probability $(p)$ using the IDL tool $r$ _correlate. We also test the dependence of each correlation with redshift using partial Spearman rank correlation analysis.

\section{Results}

\section{1 $\log \mathrm{L}_{\mathrm{X}, 200 \mathrm{~s}}-\alpha_{\mathrm{X},>200 \mathrm{~s}}$ correlation}

We see evidence for a correlation between $\log \mathrm{L}_{\mathrm{X}, 200 \mathrm{~s}}$ and $\alpha_{\mathrm{X},>200 \mathrm{~s}}$, similar to that in the optical/UV [11]. However, the presence of features in the afterglow light curves (plateaus, flares, etc.) may add scatter, potentially influence or even be the root cause of the correlation. We test these effects and other observational biases by splitting the sample and reproducing the same analyses. We report all of these tests and the final correlation in Table 1 and Fig 1.

We first test for differences between short and long duration GRBs in the $\log L_{X, 200 s}-\alpha_{X,>200 s}$ correlation. We find that long duration bursts are significantly correlated and short bursts demonstrate no significant correlation. This suggests that the correlation is related to some difference in the afterglow properties of short and long GRBs, be it their environment or jet dynamics. Therefore for all further tests of the $\log \mathrm{L}_{\mathrm{X}, 200 \mathrm{~s}}-\alpha_{\mathrm{X},>200 \mathrm{~s}}$ correlation, we exclude short GRBs.

$\mathrm{X}$-ray flares have been shown to have an internal rather than external shock origin and may be a potential source of contamination for the $\log \mathrm{L}_{\mathrm{X}, 200 \mathrm{~s}}-\alpha_{\mathrm{X},>200 \mathrm{~s}}$ correlation. Therefore we separate those afterglows with X-ray flares (without removing flaring intervals) and those without flares, and find that the two samples show very similar correlation strengths and slopes, but with slightly more scatter in the sample with flares. After excluding the flaring intervals from the light curves and refitting to obtain $\log \mathrm{L}_{\mathrm{X}, 200 \mathrm{~s}}$ and $\alpha_{\mathrm{X}, \text { avg, }>200 \mathrm{~s}}$, we find the correlation gets even tighter. Therefore, henceforth, we use the flare-removed average decay fits. 


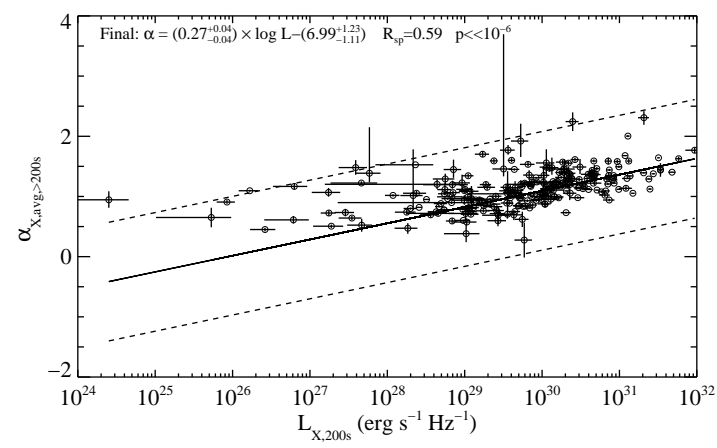

Figure 1: Final average decay - luminosity correlation using the sample that includes flare correction and those only of long duration, with corrections and sub-sample optimisation described in $\S 3$. The solid line indicates the best fit regression, and the dashed lines indicates the $2 \sigma$ deviation. This figure is reproduced from Fig. 9 of [14].

\begin{tabular}{cccccccccc}
\hline Sample & \multicolumn{2}{c}{$\begin{array}{c}\text { Parameters } \\
\mathrm{X} \text {-axis }\end{array}$} & $\mathrm{y}$-axis & $\begin{array}{c}\text { Spearman } \\
\text { Rank }\end{array}$ & $\begin{array}{c}\text { Null } \\
\text { Hypothesis }\end{array}$ & $\begin{array}{c}\text { Partial } \\
\text { Spearman } \\
\text { Rank }\end{array}$ & $\begin{array}{c}\text { Null } \\
\text { Hypothesis }\end{array}$ & $\begin{array}{c}\text { Best fit linear regression } \\
\text { Slope }\end{array}$ & $\begin{array}{c}\text { Number } \\
\text { Constant } \\
\text { in } \\
\text { Sample }\end{array}$ \\
\hline \hline Short & $\log L_{X, 200 s}$ & $\alpha_{X,>200 s}$ & -0.07 & $>0.10$ & 0.11 & $>0.10$ & $0.16_{-0.39}^{+0.10}$ & $-3.40_{-2.77}^{+11.16}$ & 9 \\
Long & $\log L_{X, 200 s}$ & $\alpha_{X,>200 s}$ & 0.59 & $\ll 10^{-6}$ & 0.59 & $\ll 10^{-6}$ & $0.27_{-0.04}^{+0.04}$ & $-6.99_{-1.10}^{+1.23}$ & 237 \\
\hline Flares & $\log L_{X, 200 s}$ & $\alpha_{X,>200 s}$ & 0.58 & $\ll 10^{-6}$ & 0.56 & $\ll 10^{-6}$ & $0.30_{-0.06}^{+0.07}$ & $-7.91_{-2.17}^{+1.84}$ & 134 \\
No Flares & $\log L_{X, 200 s}$ & $\alpha_{X,>200 s}$ & 0.59 & $\ll 10^{-6}$ & 0.64 & $\ll 10^{-6}$ & $0.28_{-0.04}^{+0.03}$ & $-7.27_{-1.01}^{+1.28}$ & 103 \\
\hline Plateau & $\log L_{X, 200 s}$ & $\alpha_{X,>200 s}$ & 0.58 & $\ll 10^{-6}$ & 0.55 & $\ll 10^{-6}$ & $0.26_{-0.06}^{+0.05}$ & $-6.81_{-1.43}^{+1.84}$ & 156 \\
No Plateau & $\log L_{X, 200 s}$ & $\alpha_{X,>200 s}$ & 0.57 & $\ll 10^{-6}$ & 0.61 & $\ll 10^{-6}$ & $0.26_{-0.05}^{+0.06}$ & $-6.82_{-1.83}^{+1.60}$ & 81 \\
\hline Final & $\log L_{X, 200 s}$ & $\alpha_{X,>200 s}$ & 0.59 & $\ll 10^{-6}$ & 0.59 & $\ll 10^{-6}$ & $0.27_{-0.04}^{+0.04}$ & $-6.99_{-1.11}^{+1.23}$ & 237 \\
\hline \hline
\end{tabular}

Table 1: Regression analysis results and correlation statistics for each sub-sample and correlation of the Xray light curve. The partial Spearman rank coefficient tests the dependence on redshift. The null hypothesis columns apply to the regular or partial Spearman rank coefficient to the left of that column. This table is adapted from Table 2 in [14].

The average decay rate is likely influenced by the complex light curve morphology of X-ray afterglows (e.g [22]). For example, a light curve with an extremely long plateau (e.g. GRB 060729, [25]) may have a shallower average decay, whereas GRBs without plateaus would be steeper. In addition $[26,27]$ have shown that there is a relationship between the time and flux of the end of the X-ray plateau, which could be another manifestation of the behaviour we have found in this study. We test the effect of X-ray plateaus on the average decay-luminosity correlation by separating our sample into those light curves that show plateau behaviour. The results in Table 1 indicate that the average decay-luminosity correlation is significant in samples of GRBs with and without plateaus. This suggests that plateaus are not solely responsible for regulating the average afterglow decay.

\subsection{Prompt emission and afterglow parameter comparison}

Upon finding a correlation between $\log \mathrm{L}_{200 s}$ and $\alpha_{>200 \mathrm{~s}}$ in both the optical/UV and X-ray afterglows, we now compare the parameters of the correlations at both wavelengths. The results are given in Table 2. Performing the analysis with the 48 GRBs found in both samples, we find the linear regressions for the correlations in both frequencies are consistent at $1 \sigma$. When swapping 


\begin{tabular}{|c|c|c|c|c|c|c|c|}
\hline \multicolumn{2}{|c|}{ Parameters } & \multirow{2}{*}{$\begin{array}{c}\text { Spearman Rank } \\
\text { Coefficient }\end{array}$} & \multirow{2}{*}{$\begin{array}{c}\text { Null } \\
\text { Hypothesis }\end{array}$} & \multirow{2}{*}{$\begin{array}{c}\text { Partial } \\
\text { Spearman Rank }\end{array}$} & \multirow{2}{*}{$\begin{array}{c}\text { Null } \\
\text { Hypothesis }\end{array}$} & \multicolumn{2}{|c|}{-Best fit linear regression- } \\
\hline$x$-axis & $y$-axis & & & & & Slope & Constant \\
\hline $\log \mathrm{L}_{\mathrm{O}, 200 \mathrm{~s}}$ & $\log \mathrm{L}_{\mathrm{X}, 200 \mathrm{~s}}$ & $0.81(0.05)$ & $5.26 \times 10^{-12}$ & 0.70 & $2.85 \times 10^{-8}$ & $0.91 \pm 0.22$ & $1.04 \pm 6.94$ \\
\hline$\alpha_{\mathrm{O},>200 \mathrm{~s}}$ & $\alpha_{\mathrm{X},>200 \mathrm{~s}}$ & $0.77(0.07)$ & $1.10 \times 10^{-10}$ & 0.75 & $1.27 \times 10^{-9}$ & $0.97 \pm 0.10$ & $0.25 \pm 0.09$ \\
\hline $\log \mathrm{L}_{\mathrm{O}, 200 \mathrm{~s}}$ & $\alpha_{\mathrm{O},>200 \mathrm{~s}}$ & $0.58(0.11)$ & $1.90 \times 10^{-5}$ & 0.50 & $2.85 \times 10^{-4}$ & $0.28 \pm 0.04$ & $-7.72 \pm 1.31$ \\
\hline $\log \mathrm{L}_{\mathrm{X}, 200 \mathrm{~s}}$ & $\alpha_{\mathrm{X},>200 \mathrm{~s}}$ & $0.69(0.09)$ & $8.03 \times 10^{-8}$ & 0.63 & $1.58 \times 10^{-6}$ & $0.26 \pm 0.05$ & $-6.71 \pm 1.39$ \\
\hline $\log \mathrm{L}_{\mathrm{O}, 200 \mathrm{~s}}$ & $\alpha_{\mathrm{X},>200 \mathrm{~s}}$ & $0.60(0.12)$ & $6.87 \times 10^{-6}$ & 0.52 & $1.53 \times 10^{-4}$ & $0.29 \pm 0.03$ & $-8.13 \pm 1.08$ \\
\hline $\log L_{X, 200 s}$ & $\alpha_{\mathrm{O},>200 \mathrm{~s}}$ & $0.65(0.10)$ & $5.58 \times 10^{-7}$ & 0.60 & $7.58 \times 10^{-6}$ & $0.32 \pm 0.06$ & $-8.70 \pm 1.68$ \\
\hline $\log E_{\text {iso }}$ & $\alpha_{\mathrm{O},>200 \mathrm{~s}}$ & & $9.05 \times 10^{-5}$ & 0.44 & $1.96 \times 10^{-3}$ & $0.21 \pm 0.05$ & $-10.22 \pm 2.57$ \\
\hline $\log E_{\text {iso }}$ & $\alpha_{\mathrm{X},>200 \mathrm{~s}}$ & $0.57(0.11)$ & $3.12 \times 10^{-5}$ & 0.47 & $8.70 \times 10^{-4}$ & $0.21 \pm 0.04$ & $-9.60 \pm 2.16$ \\
\hline $\log E_{\text {iso }}$ & $\log L_{0,200 s}$ & $0.76(0.06)$ & $4.51 \times 10^{-10}$ & 0.66 & $4.59 \times 10^{-7}$ & $1.09 \pm 0.13$ & $-25.27 \pm 6.92$ \\
\hline $\log \mathrm{E}_{\text {iso }}$ & $\log \mathrm{L}_{\mathrm{X}, 200 \mathrm{~s}}$ & $0.83(0.05)$ & $5.04 \times 10^{-13}$ & 0.76 & $4.78 \times 10^{-10}$ & $1.10 \pm 0.15$ & $-27.81 \pm 7.89$ \\
\hline $\log E_{\text {peak }}$ & $\alpha_{\mathrm{O},>200 \mathrm{~s}}$ & $0.45(0.13)$ & $2.05 \times 10^{-3}$ & 0.38 & $1.20 \times 10^{-2}$ & $0.48 \pm 0.17$ & $-0.22 \pm 0.41$ \\
\hline $\log E_{\text {peak }}$ & $\alpha_{\mathrm{X},>200 \mathrm{~s}}$ & $0.48(0.13)$ & $9.22 \times 10^{-4}$ & 0.40 & $7.52 \times 10^{-3}$ & $0.48 \pm 0.15$ & $0.03 \pm 0.36$ \\
\hline $\log E_{\text {peak }}$ & $\log \mathrm{L}_{\mathrm{O} \times 200 \mathrm{~s}}$ & $0.66(0.11)$ & $1.16 \times 10^{-6}$ & 0.58 & $3.51 \times 10^{-5}$ & $2.97 \pm 0.76$ & $24.53 \pm 1.95$ \\
\hline $\log \mathrm{E}_{\text {peak }}$ & $\log L_{X, 200 s}$ & $0.75(0.10)$ & $4.74 \times 10^{-9}$ & 0.70 & $1.38 \times 10^{-7}$ & 2.970 .67 & $22.50 \pm 1.73$ \\
\hline
\end{tabular}

Table 2: For each pair of parameters examined, this table contains: the Spearman rank correlation coefficient with its associated null hypothesis; the coefficient of the partial Spearman rank with its associated null hypothesis; the slope and constant values provided by the best fit linear regression. We also provide the $1 \sigma$ error of the Spearman rank coefficient. Table is a reproduction of Table 2 from [15].

the X-ray and optical/UV luminosity and decay parameters, i.e $\log \mathrm{L}_{\mathrm{O}, 200 \mathrm{~s}}$ versus $\alpha_{\mathrm{X},>200 \mathrm{~s}}$ and $\log \mathrm{L}_{\mathrm{X}, 200 \mathrm{~s}}$ versus $\alpha_{\mathrm{O},>200 \mathrm{~s}}$ similar strength relationships are found. This supports the validity of the luminosity-decay correlation. Strong correlations are also observed when correlating the luminosities in both frequencies and the decay indices in both frequencies.

In Table 2 we also provide the results of the comparison of the parameters of the optical/UV and X-ray luminosity-decay correlations with the prompt emission parameters: $\log \mathrm{E}_{\text {iso }}$ and $\mathrm{E}_{\text {peak }}$. Comparison of the optical/UV and X-ray luminosity with $\log \mathrm{E}_{\text {iso }}$ indicates strong correlations and the linear regressions are consistent to within $1 \sigma$ error. We also provide the results of the comparison of $\log \mathrm{E}_{\text {iso }}$ with $\alpha_{\mathrm{O},>200 \mathrm{~s}}$ and $\alpha_{\mathrm{X},>200 \mathrm{~s}}$. These correlations are slightly less strong compared to that found between luminosity and $\log \mathrm{E}_{\mathrm{iso}}$, but within errors the equations for the linear regression for both the optical/UV and X-ray $\alpha_{>200 s}$ against $\log \mathrm{E}_{\text {iso }}$ are consistent with each other.

The Amati relation indicates a relationship between $\mathrm{E}_{\text {iso }}$ and $\mathrm{E}_{\text {peak }}$ [1]. Therefore we may already predict correlations between $\mathrm{E}_{\text {peak }}$ and the afterglow parameters, but for completeness we report the strength of these correlations. For $\log \mathrm{E}_{\text {peak }}$ against $\log \mathrm{L}_{\mathrm{O}, 200 \mathrm{~s}}$ and $\log \mathrm{L}_{\mathrm{X}, 200 \mathrm{~s}}$, we notice that the Spearman rank coefficient is smaller than that found for $\log \mathrm{L}_{200 \mathrm{~s}}$ with $\log \mathrm{E}_{\mathrm{iso}}$, indicating that the relationships involving the prompt emission peak energy are weaker in comparison to the relationships observed with the isotropic energy; consistent with that found by [6]. Also for $\log \mathrm{E}_{\text {peak }}$ against $\alpha_{\mathrm{O},>200 \mathrm{~s}}$ and $\alpha_{\mathrm{X},>200 \mathrm{~s}}$, the Spearman rank coefficients are smaller in comparison to the Spearman rank coefficients found for the correlations between the decay indices and $\log \mathrm{E}_{\mathrm{iso}}$.

\section{Discussion}

We have shown that the $\log \mathrm{L}_{200 \mathrm{~s}}-\alpha_{>200 \mathrm{~s}}$ correlation, observed in the optical/UV light curves by [11], is also observed in the X-ray light curves, suggesting that the brightest afterglows decay 
more quickly than the fainter afterglows. This points towards a common underlying mechanism producing both the X-ray and optical/UV afterglows. We can therefore generally exclude models that invoke different emission mechanisms that separately produce the X-ray and optical/UV afterglow.

Pre-Swift observations of late time X-ray afterglows also seemed to suggest the brightest X-ray afterglows decay more quickly than fainter afterglows [28, 29, 30], but a larger sample including some of the first Swift X-ray light curves [31] was not able to support previous claims (see also [14]). In this analysis, the correlation between luminosity and temporal behaviour is investigated at a much earlier time, when there is greater spread in the luminosity distribution, and the average decay index is determined using almost the entire observed afterglow.

We also have shown that the X-ray and optical/UV $\log \mathrm{L}_{200 \text { s }}$ are correlated with $\log \mathrm{E}_{\text {iso }}$ and $E_{\text {peak. }}$ This is consistent with previous studies (e.g. [29, 32, 33, 34]), in particular [6] and [35], who performed a similar study using early X-ray luminosity, $5-10$ minutes after trigger. We have also shown that the optical/UV and X-ray $\alpha_{>200 \text { s }}$ are correlated with $\log \mathrm{E}_{\text {iso }}$ and $\mathrm{E}_{\text {peak }}$. Altogether, the correlations we report indicate that the GRBs with the brightest, fastest, decaying afterglows also have the largest observed prompt emission energies and typically larger peak spectral energy.

We now investigate if these observations are consistent with the predictions of the standard afterglow model. This model provides different relationships between parameters depending on the ordering of the spectral frequencies. Therefore it is necessary to perform a Monte Carlo simulation to obtain the expected relationships between various parameters for a sample of 48 GRBs. Using $10^{4}$ trials, we simulated the optical/UV (at $1600 \AA$ ) and X-ray (at $1 \mathrm{keV}$ ) flux densities for 48 GRBs using equation 8 of [36] and equations 4,5 and 6 given in [37] for $F_{v, \max }, v_{m}$ and $v_{c}$. In this simulation we assume that all GRBs are produced in a constant density medium. To compute $F_{v, \max }, v_{m}$ and $v_{c}$ we needed to provide values for the microphysical parameters. These were selected at random from log-normal distributions which had $3 \sigma$ intervals ranging between: 0.01 0.3 for the fraction of energy given to the electrons, $\varepsilon_{e} ; 5 \times 10^{-4}-0.5$ for the fraction of energy given to the magnetic field, $\varepsilon_{B}$, and $10^{-3}-10^{3} \mathrm{~cm}^{-2}$ for the density of the external medium. The centre of each of these distributions is at the logarithmic midpoint. For the electron energy index $p$, we centred the distribution at 2.4 , as determined by [38], however, we set the $1 \sigma$ width to be 0.2 rather than 0.59 . Since the closure relations fail for $p$ values $<2$, we re-sampled the $p$ value when $p<2$ was selected. The value of $p$ along with the position of $v_{c}$ relative to the observed band and redshift (selected from a uniform distribution with the range $0.5-4.5$, a similar range as the observed sample), dictate the values of $\alpha, \beta$ and the k-correction (as given in [39]).

For the 48 GRBs in each trial, we selected a prompt emission energy from a log-normal distribution with a $3 \sigma$ range $10^{51}-10^{54} \mathrm{erg}$; a range similar to that of the GRBs in this sample. We picked a random value between $10 \%$ and $99 \%$ for the efficiency, which we used to convert the prompt emission energy into kinetic energy. Once all the microphysical parameters, redshift and kinetic energy had been selected, we were then able to determine the position of $v_{c}$ and thus knew where it was in relation to $v_{O}$ and $v_{X}$. With this information, we then calculated the value of the optical/UV and X-ray fluxes and converted these to luminosity. As a byproduct of calculating the optical/UV and X-ray luminosities, we also have simulated distributions for $\mathrm{E}_{\text {iso }}$ and $\alpha$. Therefore we also produce predictions for comparisons that involve these parameters. For the parameters of 48 GRBs in each trial, we performed linear regression using the IDL routine sixlin, and we also 


\begin{tabular}{llccc}
\hline \multicolumn{2}{c}{ Parameters } & $\begin{array}{c}\text { Simulated Spearman } \\
\text { x-axis }\end{array}$ & $y$-axis & \multicolumn{2}{c}{$\begin{array}{c}\text { Best fit linear regression for simulation- } \\
\text { Rank Coefficient }\end{array}$} & Slope & Constant \\
\hline \hline $\log \mathrm{L}_{\mathrm{O}, 200 \mathrm{~s}}$ & $\log \mathrm{L}_{\mathrm{X}, 200 \mathrm{~s}}$ & $0.92 \pm 0.0$ & $0.82 \pm 0.0$ & $3.76 \pm 1.25$ \\
$\alpha_{\mathrm{O},>200 \mathrm{~s}}$ & $\alpha_{\mathrm{X},>200 \mathrm{~s}}$ & $0.74 \pm 0.0$ & $1.10 \pm 0.1$ & $0.04 \pm 0.17$ \\
$\log \mathrm{L}_{\mathrm{O}, 200 \mathrm{~s}}$ & $\alpha_{\mathrm{O},>200 \mathrm{~s}}$ & $0.30 \pm 0.14$ & $0.04 \pm 0.02$ & $-0.31 \pm 0.65$ \\
$\log \mathrm{L}_{\mathrm{X}, 200 \mathrm{~s}}$ & $\alpha_{\mathrm{X},>200 \mathrm{~s}}$ & $0.20 \pm 0.14$ & $0.04 \pm 0.03$ & $0.10 \pm 0.78$ \\
$\log \mathrm{E}_{\text {iso }}$ & $\alpha_{\mathrm{O},>200 \mathrm{~s}}$ & $0.06 \pm 0.15$ & $0.03 \pm 0.06$ & $-0.32 \pm 2.91$ \\
$\log \mathrm{E}_{\text {iso }}$ & $\alpha_{\mathrm{X},>200 \mathrm{~s}}$ & $0.09 \pm 0.15$ & $0.04 \pm 0.06$ & $-0.76 \pm 3.13$ \\
$\log \mathrm{E}_{\text {iso }}$ & $\log \mathrm{L}_{\mathrm{O}, 200 \mathrm{~s}}$ & $0.51 \pm 0.11$ & $4.43 \pm 1.03$ & $-200.76 \pm 54.10$ \\
$\log \mathrm{E}_{\text {iso }}$ & $\log \mathrm{L}_{\mathrm{X}, 200 \mathrm{~s}}$ & $0.54 \pm 0.11$ & $3.28 \pm 0.71$ & $-142.22 \pm 37.33$ \\
\hline
\end{tabular}

Table 3: The Spearman rank coefficient and linear regression parameters as predicted by the synchrotron model for a sample of 48 GRBs. These values were computed with a Monte Carlo simulation with $10^{4}$ trials. Table is a reproduction of Table 1 from [15].

calculated the Spearman rank coefficient. The results of the simulation can be found in Table 3 .

Since the standard afterglow model predicts the optical/UV and X-ray emission produced by the same mechanism from an isotropic outflow, we would expect to see relationships between $\log \mathrm{L}_{\mathrm{O}, 200 \mathrm{~s}} \& \log \mathrm{L}_{\mathrm{X}, 200 \mathrm{~s}}$ and $\alpha_{\mathrm{O},>200 \mathrm{~s}}$ versus $\alpha_{\mathrm{X},>200 \mathrm{~s}}$. Our observed relationships between these parameters can therefore be explained easily by the standard afterglow model and are fully consistent with the simulations. A relationship between $\log \mathrm{E}_{\text {iso }}$ and $\log \mathrm{L}_{200 \mathrm{~s}}$ is also expected in the standard afterglow model, but the comparison of our observed relationship to the simulations suggests that the observed linear regression slope is less steep than predicted by the simulation. Furthermore, the relationships we observe, between $\log \mathrm{L}_{200 \mathrm{~s}}$ and $\alpha_{>200 \mathrm{~s}}$, and $\log \mathrm{E}_{\text {iso }}$ and $\alpha_{>200 \mathrm{~s}}$, are not expected in the standard afterglow model and are not predicted by the simulations.

Since the standard afterglow does not succeed in fully predicting all of our observed correlations, it is likely that a more complex outflow model is required. This conclusion is similar to that drawn during the separate investigation of the optical/UV $\log L_{200 s}-\alpha_{>200 s}$ decay correlation [11].

\subsection{Alternative Models}

There are three main possibilities that could make the outflow complex enough to be able to reproduced the observed correlations. The first is that perhaps there is some mechanism or parameter that controls the amount of energy given to and distributed during the prompt and afterglow phases and that also regulates the afterglow decay rate. This should occur in such a way that for events with the largest gamma-ray isotropic energy, the energy given to the afterglow is released quickly, resulting in an initially bright afterglow which decays rapidly. Conversely, if the gammaray isotropic energy is smaller, then the afterglow energy is released slowly over a longer period, the afterglow will be less bright initially and decay at a slower rate.

The second possibility is that the correlations could be a geometric effect, perhaps the result of the observer's viewing angle. Jets viewed away from the jet-axis may have fainter afterglows that decay less quickly in comparison to afterglows viewed closer to the centre of the jet (see Fig 


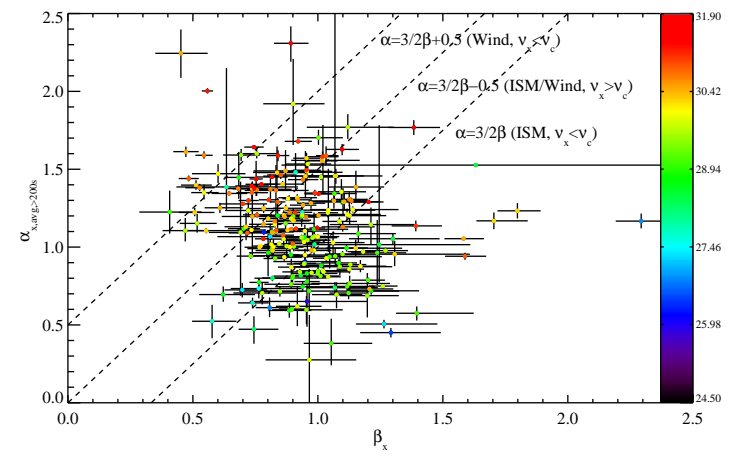

Figure 2: The average temporal decay $\left(\alpha_{\mathrm{X},>200 \mathrm{~s}}\right)$ and average spectral energy index $\left(\beta_{\mathrm{X}}\right)$ are compared with $\log L_{X, 200 s}$ (colour scale), demonstrating consistency and trends with the closure relations (dashed lines). The high luminosity (redder) points are roughly consistent with wind-like environments. This figure is reproduced from Fig. 15 of [14].

3. of [4]). Similarly, this will also affect the observed prompt emission, with jets viewed off-axis appearing to have lower isotropic energy and lower peak spectral energy [40].

The third possibility could be related to the circumburst environment. The closure relations relate $\alpha$ and the spectral index $\beta$ through different relationships depending on the ordering of the synchrotron spectral parameters and the density profile or the external medium. If the correlation is affected by the circumburst environment, we expect to see GRBs with the highest luminosities favouring a particular environment. No apparent correlation is observed in the optical/UV [11]. However in the X-ray (see Figure 2), the highest luminosity GRBs tend toward the lines demarcating the $r^{-2}$ wind environment. The ambiguity in the $v_{x}>v_{c}$ cases prohibits us from making a strong statement on the role of circumburst environment, but it may be another possible contribution in that the initially brightest GRB afterglows may be more likely to live in wind-like environments (see also [41]).

\section{Conclusions}

This proceeding has summarised the work presented in [14] and [15]. We have shown that the correlation between luminosity (measured at restframe $200 \mathrm{~s} ; \log \mathrm{L}_{200 \mathrm{~s}}$ ) and average decay rate (measured from $200 \mathrm{~s} ; \alpha_{>200 \mathrm{~s}}$ ) is observed in the X-ray light curve sample as well as the optical/UV [11]. When we rerun the correlations with the same GRBs we find the luminosity-decay correlations are consistent. This suggests a single underlying mechanism producing the correlations in both bands and it is not dependent on their detailed temporal behaviour. We also show significant correlations between the logarithmic optical/UV and X-ray luminosity $\left(\log \mathrm{L}_{\mathrm{O}, 200 \mathrm{~s}}, \log \mathrm{L}_{\mathrm{X}, 200 \mathrm{~s}}\right)$ and the optical/UV and X-ray decay indices $\left(\alpha_{\mathrm{O},>200 \mathrm{~s}}\right.$ and $\left.\alpha_{\mathrm{X},>200 \mathrm{~s}}\right)$ and all four of these parameters are found to be correlated with the prompt emission parameters: isotropic energy $\left(\mathrm{E}_{\mathrm{iso}}\right)$ and restframe peak spectral energy $\left(E_{\text {peak }}\right)$. Together these correlations imply that the GRBs with the brightest afterglows in the X-ray and optical/UV bands, decay the fastest and they also have the largest observed prompt emission energies and typically larger peak spectral energy. This suggests that what happens during the prompt phase has direct implications on the afterglow. 
We used a Monte Carlo simulation to examine whether the standard afterglow model is able to explain the observed correlations. Overall, observed correlations between the luminosities in both the X-ray and optical/UV bands and between the luminosities and the isotropic energy are consistent with the predictions of the simulation. However, observed relationships involving the average decay indices with either luminosity at $200 \mathrm{~s}$ or the isotropic $\gamma$-ray energy are not consistent with the simulation. We therefore suggest that a more complex afterglow or outflow model is required to produce all the observed correlations. This may be due to either a viewing angle effect or by some mechanism or physical property controlling the energy release within the outflow. The environment in which the GRB exploded may also contribute to the observed correlation.

\section{Acknowledgements}

This research has made use of data obtained from the High Energy Astrophysics Science Archive Research Center (HEASARC) and the UK Swift Science Data Centre provided by NASA's Goddard Space Flight Center and the University of Leicester, UK, respectively. SRO gratefully acknowledges the support of the Leverhulme Trust Early Career Fellowship. MJP, AAB, NPMK and PJS acknowledge the support of the UK Space Agency.

\section{References}

[1] L. Amati, F. Frontera, M. Tavani, J. J. M. in’t Zand, A. Antonelli, E. Costa et al., Intrinsic spectra and energetics of BeppoSAX Gamma-Ray Bursts with known redshifts, Astron. Astrophys. 390 (2002) 81 [arXiv:astro-ph/0205230].

[2] G. Ghirlanda, G. Ghisellini and D. Lazzati, The Collimation-corrected Gamma-Ray Burst Energies Correlate with the Peak Energy of Their vF Spectrum, Astrophys. J. 616 (2004) 331 [arXiv:astro-ph/0405602].

[3] M. G. Dainotti, V. F. Cardone and S. Capozziello, A time-luminosity correlation for $\gamma$-ray bursts in the X-rays, Mon. Not. R. Astr. Soc. 391 (2008) L79 [0 809.1389 ].

[4] A. Panaitescu and W. T. Vestrand, Taxonomy of gamma-ray burst optical light curves: identification of a salient class of early afterglows, Mon. Not. R. Astr. Soc. 387 (2008) 497 [0803 . 1872].

[5] M. G. Bernardini, R. Margutti, J. Mao, E. Zaninoni and G. Chincarini, The X-ray light curve of gamma-ray bursts: clues to the central engine, Astron. Astrophys. 539 (2012) A3 [1112.1058].

[6] P. D’Avanzo, R. Salvaterra, B. Sbarufatti, L. Nava, A. Melandri, M. G. Bernardini et al., A complete sample of bright Swift Gamma-ray bursts: X-ray afterglow luminosity and its correlation with the prompt emission, Mon. Not. R. Astr. Soc. 425 (2012) 506 [1206.2357].

[7] L. Li, E.-W. Liang, Q.-W. Tang, J.-M. Chen, S.-Q. Xi, H.-J. Lü et al., A Comprehensive Study of Gamma-Ray Burst Optical Emission. I. Flares and Early Shallow-decay Component, Astrophys. J. 758 (2012) 27 [1203.2332].

[8] E.-W. Liang, L. Li, H. Gao, B. Zhang, Y.-F. Liang, X.-F. Wu et al., A Comprehensive Study of Gamma-Ray Burst Optical Emission. II. Afterglow Onset and Late Re-brightening Components, Astrophys. J. 774 (2013) 13 [1210.5142]. 
[9] E. Zaninoni, M. G. Bernardini, R. Margutti, S. Oates and G. Chincarini, Gamma-ray burst optical light-curve zoo: comparison with X-ray observations, Astron. Astrophys. 557 (2013) A12 [1303.6924].

[10] A. Panaitescu, W. T. Vestrand and P. Woźniak, Peaks of optical and X-ray afterglow light curves, Mon. Not. R. Astr. Soc. 433 (2013) 759 [1305. 0809].

[11] S. R. Oates, M. J. Page, M. De Pasquale, P. Schady, A. A. Breeveld, S. T. Holland et al., A correlation between the intrinsic brightness and average decay rate of Swift/UVOT gamma-ray burst optical/ultraviolet light curves, Mon. Not. R. Astr. Soc. 426 (2012) L86 [1208 . 1856].

[12] P. W. A. Roming, T. E. Kennedy, K. O. Mason, J. A. Nousek, L. Ahr, R. E. Bingham et al., The Swift Ultra-Violet/Optical Telescope, Space Science Reviews 120 (2005) 95 [arXiv:astro-ph/0507413].

[13] N. Gehrels, G. Chincarini, P. Giommi, K. O. Mason, J. A. Nousek, A. A. Wells et al., The Swift Gamma-Ray Burst Mission, Astrophys. J. 611 (2004) 1005.

[14] J. L. Racusin, S. R. Oates, M. de Pasquale and D. Kocevski, A Correlation between the Intrinsic Brightness and Average Decay Rate of Gamma-Ray Burst X-Ray Afterglow Light Curves, Astrophys. J. 826 (2016) 45 [1605.00719].

[15] S. R. Oates, J. L. Racusin, M. De Pasquale, M. J. Page, A. J. Castro-Tirado, J. Gorosabel et al., Exploring the canonical behaviour of long gamma-ray bursts using an intrinsic multiwavelength afterglow correlation, Mon. Not. R. Astr. Soc. 453 (2015) 4121 [1508. 06567 ].

[16] D. N. Burrows, J. E. Hill, J. A. Nousek, J. A. Kennea, A. Wells, J. P. Osborne et al., The Swift X-Ray Telescope, Space Science Reviews 120 (2005) 165 [arXiv: astro-ph/ 0508071 ].

[17] P. A. Evans, A. P. Beardmore, K. L. Page, L. G. Tyler, J. P. Osborne, M. R. Goad et al., An online repository of Swift/XRT light curves of $\gamma$-ray bursts, Astron. Astrophys. 469 (2007) 379 [0704.0128].

[18] P. A. Evans, A. P. Beardmore, K. L. Page, J. P. Osborne, P. T. O’Brien, R. Willingale et al., Methods and results of an automatic analysis of a complete sample of Swift-XRT observations of GRBs, Mon. Not. R. Astr. Soc. 397 (2009) 1177 [0812.3662].

[19] P. W. A. Roming, T. S. Koch, S. R. Oates, B. L. Porterfield, A. J. Bayless, A. A. Breeveld et al., A Large Catalog of Homogeneous Ultra-Violet/Optical GRB Afterglows: Temporal and Spectral Evolution, Astrophys. J. Suppl. 228 (2017) 13 [1701.03713].

[20] S. R. Oates, M. J. Page, P. Schady, M. De Pasquale, T. S. Koch, A. A. Breeveld et al., A statistical study of gamma-ray burst afterglows measured by the Swift Ultraviolet Optical Telescope, Mon. Not. R. Astr. Soc. 395 (2009) 490 [0901.3597].

[21] P. Schady, M. J. Page, S. R. Oates, M. Still, M. de Pasquale, T. Dwelly et al., Dust and metal column densities in gamma-ray burst host galaxies, Mon. Not. R. Astr. Soc. 401 (2010) 2773 [0910 . 2590].

[22] J. L. Racusin, E. W. Liang, D. N. Burrows, A. Falcone, T. Sakamoto, B. B. Zhang et al., Jet Breaks and Energetics of Swift Gamma-Ray Burst X-Ray Afterglows, Astrophys. J. 698 (2009) 43 [0812.4780].

[23] J. L. Racusin, S. R. Oates, P. Schady, D. N. Burrows, M. de Pasquale, D. Donato et al., Fermi and Swift Gamma-ray Burst Afterglow Population Studies, Astrophys. J. 738 (2011) 138 [1106.2469]. 
[24] B. Zhang, Y. Z. Fan, J. Dyks, S. Kobayashi, P. Mészáros, D. N. Burrows et al., Physical Processes Shaping Gamma-Ray Burst X-Ray Afterglow Light Curves: Theoretical Implications from the Swift X-Ray Telescope Observations, Astrophys. J. 642 (2006) 354.

[25] D. Grupe, C. Gronwall, X. Wang, P. W. A. Roming, J. Cummings, B. Zhang et al., Swift and XMM-Newton Observations of the Extraordinary Gamma-Ray Burst 060729: More than 125 Days of X-Ray Afterglow, Astrophys. J. 662 (2007) 443 [arXiv: astro-ph/ 0611240 ].

[26] M. G. Dainotti, R. Willingale, S. Capozziello, V. Fabrizio Cardone and M. Ostrowski, Discovery of a Tight Correlation for Gamma-ray Burst Afterglows with "Canonical” Light Curves, Astrophys. J. Letters 722 (2010) L215 [1009.1663].

[27] M. G. Dainotti, V. Petrosian, J. Singal and M. Ostrowski, Determination of the Intrinsic Luminosity Time Correlation in the X-Ray Afterglows of Gamma-Ray Bursts, Astrophys. J. 774 (2013) 157 [1307. 7297].

[28] M. Boër and B. Gendre, Evidences for two Gamma-Ray Burst afterglow emission regimes, Astron. Astrophys. 361 (2000) L21 [astro-ph/0008385].

[29] C. Kouveliotou, S. E. Woosley, S. K. Patel, A. Levan, R. Blandford, E. Ramirez-Ruiz et al., Chandra Observations of the X-Ray Environs of SN 1998bw/GRB 980425, Astrophys. J. 608 (2004) 872 [astro-ph/0401184].

[30] B. Gendre and M. Boër, Decay properties of the X-ray afterglows of gamma-ray bursts, Astron. Astrophys. 430 (2005) 465 [astro-ph / 0411238 ].

[31] B. Gendre, A. Galli and M. Boër, X-Ray Afterglow Light Curves: Toward A Standard Candle?, Astrophys. J. 683 (2008) 620.

[32] M. De Pasquale, L. Piro, B. Gendre, L. Amati, L. A. Antonelli, E. Costa et al., The BeppoSAX catalog of GRB X-ray afterglow observations, Astron. Astrophys. 455 (2006) 813 [arXiv:astro-ph/0507708].

[33] M. Nysewander, A. S. Fruchter and A. Pe'er, A Comparison of the Afterglows of Short-and Long-duration Gamma-ray Bursts, Astrophys. J. 701 (2009) 824 [0 806.3607$].$

[34] D. A. Kann, S. Klose, B. Zhang, D. Malesani, E. Nakar, A. Pozanenko et al., The Afterglows of Swift-era Gamma-ray Bursts. I. Comparing pre-Swift and Swift-era Long/Soft (Type II) GRB Optical Afterglows, Astrophys. J. 720 (2010) 1513 [0712 .2186].

[35] R. Margutti, E. Zaninoni, M. G. Bernardini, G. Chincarini, F. Pasotti, C. Guidorzi et al., The prompt-afterglow connection in gamma-ray bursts: a comprehensive statistical analysis of Swift X-ray light curves, Mon. Not. R. Astr. Soc. 428 (2013) 729 [1203. 1059 ].

[36] R. Sari, T. Piran and R. Narayan, Spectra and Light Curves of Gamma-Ray Burst Afterglows, Astrophys. J. Letters 497 (1998) L17 [arXiv : astro-ph/9712005].

[37] B. Zhang, E. Liang, K. L. Page, D. Grupe, B.-B. Zhang, S. D. Barthelmy et al., GRB Radiative Efficiencies Derived from the Swift Data: GRBs versus XRFs, Long versus Short, Astrophys. J. 655 (2007) 989 [astro-ph/0610177].

[38] P. A. Curran, R. L. C. Starling, A. J. van der Horst and R. A. M. J. Wijers, Testing the blast wave model with Swift GRBs, Mon. Not. R. Astr. Soc. 395 (2009) 580.

[39] E. Berger, S. R. Kulkarni and D. A. Frail, A Standard Kinetic Energy Reservoir in Gamma-Ray Burst Afterglows, Astrophys. J. 590 (2003) 379 [astro-ph / 0301268 ]. 
[40] E. Ramirez-Ruiz, J. Granot, C. Kouveliotou, S. E. Woosley, S. K. Patel and P. A. Mazzali, An Off-Axis Model of GRB 031203, Astrophys. J. Letters 625 (2005) L91 [arXiv: astro-ph/ 0412145 ].

[41] M. De Pasquale, S. Schulze, D. A. Kann, S. Oates and B. Zhang, Physical properties of rapidly decaying Afterglows, in EAS Publications Series, A. J. Castro-Tirado, J. Gorosabel and I. H. Park, eds., vol. 61 of EAS Publications Series, pp. 217-221, July, 2013, DOI.

\section{DISCUSSION}

JIM BEALL: You mentioned 'circumburst' winds. If these are associated with massive stars would one expect particular stellar wind profiles. Can you comment on this?

SAM OATES: The analytical expressions describing GRBs in wind-like media assume simple density profiles $r^{-k}$, with either $k=0$, a constant density profile or $k=2$ a stellar wind-type profile. In general observations don't allow a more accurate determination of $k$ and are more frequently shown to prefer a constant density circumstellar density profile over a wind profile.

MARKUS BÖTTCHER: A Spearman rank of $\sim 0.6$ is not usually considered a strong correlation. The p-value depends on the assumed distributions of $\alpha$ and $\mathrm{L}$. What are those distributions used in the Monte Carlo simulations?

SAM OATES: We have run two separate Monte Carlo simulations, one to test if the optical/UV correlation could be due to chance or be a result of selection effects and another to test if the observed correlations are consistent with the predictions of the standard afterglow model.

In the first Monte Carlo simulation, performed in [11], we simulated a distribution of 48 pairs of $\log \mathrm{L}_{\mathrm{O}, 200 \mathrm{~s}}-\alpha_{\mathrm{O},>200 \mathrm{~s}}$ data points selected at random from linear distributions of $\log \mathrm{L}_{\mathrm{O}, 200 \mathrm{~s}}$ and $\alpha_{\mathrm{O},>200 \mathrm{~s}}$, which have the same ranges as the observed sample. For each pair of values, we produced a simulated observer frame light curve to check if the light curve would have met the selection criteria, discarding and redrawing a new pair of $\log \mathrm{L}_{\mathrm{O}, 200 \mathrm{~s}}$ and $\alpha_{\mathrm{O},>200 \mathrm{~s}}$ when the selection criteria wasn't met and repeating this until 48 simulated pairs were obtained. We then ran the Spearman rank correlation on each set of simulated data points. We repeated this $10^{6}$ times. We found our correlation is not due to chance or a result of selection effects at $4.1 \sigma$ confidence.

For the second Monte Carlo simulation, to see if our observations were consistent with the predictions of the standard afterglow model, we simulated luminosity at restframe $200 \mathrm{~s}$ and $\alpha$ values using the equations for flux density and the closure relations as given in the standard afterglow model. The flux density depends on the energy of the GRB and the microphysical parameters. The prompt emission energy was chosen from a log-normal distribution similar to that of the GRBs in this sample whereas the microphysical parameters were selected from log-normal distributions based on values found for GRBs in the literature. The $\alpha$ values were obtained from the appropriate closure relation, which is determined by ordering of the observed frequency with respect to the simulated synchrotron peak frequency and the synchrotron cooling frequency. 